\title{
Flocking Regimes in a Simple Lattice Model
}

\author{
J. R. Raymond and M. R. Evans \\ SUPA, School of Physics, University of Edinburgh,Mayfield Road, Edinburgh EH9 3JZ, UK
}

April 19, 2018

\begin{abstract}
We study a one-dimensional lattice flocking model incorporating all three of the flocking criteria proposed by Reynolds [Computer Graphics 214 (1987)]: alignment, centring and separation. The model generalises that introduced by O. J. O' Loan and M. R. Evans J. Phys. A. 32 L99 (1999). We motivate the dynamical rules by microscopic sampling considerations. The model exhibits various flocking regimes: the alternating flock, the homogeneous flock and dipole structures. We investigate these regimes numerically and within a continuum mean-field theory.
\end{abstract}

Pacs: 05.70.Fh 64.60.Cn 87.10e

\section{Introduction}

Flocking refers to a family of behaviours regularly seen in nature, including schooling of fish [1] and flocking of birds and moths 2. Whilst the features that are truly essential to flocks remain open to speculation [3, 4, 5, 6] it is clear that the variety of biological[7, 1, 2] and engineered [8] systems in which flocking behaviour does, or could, prove useful is vast. Statistical mechanics has recently played an important role in uncovering and determining the source of many flocking phenomena $[9]$.

One of the pioneers in flocking research was Reynolds 3 . Within his work he determined three behaviours characteristic of flocking: the desire to match the velocity of flockmates (alignment), the desire to stay close to flockmates (centring), and the desire to avoid collisions (separation). Although Reynolds' original research was motivated by applications in computer graphics, his definitions have been adopted widely and implemented by researchers from a variety of disciplines. Reynolds coined the term boids to describe the generic selfpropelled particles obeying these rules, and we shall continue that convention in this paper.

Within the physical science literature research has focused primarily on the steady state behaviour of flocking models and the non-equilibrium elements which cause solutions to differ from equilibrium systems. Many models involving only an alignment interaction have been shown to demonstrate a symmetry broken velocity state[10]. Results have also been found 
which are strongly non-equilibrium in one and two dimensions, where equilibrium systems do not undergo comparable symmetry breaking phase transitions 12]. Important work on alignment has included that of Vicsek et al[10] and Toner and Tu[11] who shed light on the non-equilibrium processes involved. A wide variety of models also exist in two or three dimensions incorporating one or several of Reynolds' rules besides alignment [13, 14. The lattice model of [14], which includes separation and alignment, shows a density-dependent symmetry broken state, for example. Similar results have also been derived using a network approach to flocking, whereby boids are nodes within a dynamic graph 15 .

There are fewer obvious physical realisations of one-dimensional flocking, although analogy may be made with ring shaped aquariums, and with pedestrian dynamics in corridors 16. However, research into one-dimensional systems certainly is interesting from a fundamental viewpoint: in particular, nonequilibrium phase transitions may be exhibited [17. In the one dimension alignment model of Czirok et al 18] and the one dimensional lattice model of O'Loan and Evans [19] novel states not anticipated by higher dimensional treatments were found. In [18] a symmetry broken steady state was reported, whilst [19] found a steady state which alternated its direction of motion on an unusually short time scale $O(\ln N)$ where $N$

is the number of boids in the system. In the one dimensional model of Levine et al 21] the effects of centring and separation were considered and new behaviours were also found.

Our aim in this work is to introduce and study a one-dimensional lattice model involving all three of Reynolds' flocking criteria. We do this by generalising the model of [19]. We demonstrate a variety of new flocking regimes: further to the alternating flock state found in [19] we show that on small systems a homogeneous flock may occur; also, the inclusion of centring may produce various dipole structures in which the velocities of the boids point towards the centre of the structure. Our generalisation also addresses some deficiencies in the dynamics of [19] which implied full knowledge of neighbouring boids - in the present work the dynamics is motivated from the sampling of a finite number of neighbouring boids and yields a smoother form [Eq. (6) below] for the expected update velocity of a boid as a function of the average neighbourhood velocity.

The paper is organised as follows. In section 2 we define and motivate the model to be studied. In section 3 we present numerical simulations of the model and examine the different regimes that may be observed. In section 4 we employ mean-field theory to provide further evidence for these regimes. We conclude with a discussion in section 5 .

\section{Boid Dynamics}

\subsection{Model synopsis}

We first review the model of [19] which consists of $N$ boids inhabiting a lattice of $L$ sites with unit lattice spacing. The global density, $\langle\rho\rangle$, is defined as $\langle\rho\rangle=N / L$. Each boid $\mu$ is defined by a position $x_{\mu}=1 \ldots L$ and velocity $v_{\mu}= \pm 1$. An update of the system consists of the following steps:

1. A boid is chosen at random. 
2. A preferred direction $U\left(x_{\mu}, t\right)= \pm 1$ or 0 is determined from the velocity and spatial distribution of neighbouring boids.

3. $v_{\mu}$ is updated to \pm 1 with probability $W_{ \pm 1}$

$$
W_{ \pm 1}=1 / 2 \pm(1 / 2-\eta) U\left(x_{\mu}, t\right)
$$

where the constant $\eta$ is a parameter of the model.

4. The boid moves: $x_{\mu}$ is updated to $x_{\mu}+v_{\mu}$.

We take the neighbourhood of site $i$ to be the sites $i-1, i$ and $i+1$. In the model of [19] $U$ is given by the local majority velocity which we now define. Let the number of boids on a site with velocity $v$ be $n_{v}(x, t)$, the site density $\rho(x, t)=\sum_{v} n_{v}(x, t)$ and site momentum $\phi(x, t)=\sum_{v} v n_{v}(x, t)$. The majority velocity of neighbouring boids is equivalent to the sign of the neighbourhood (3 site) averaged momentum $\widehat{\phi}(x, t)$ given by

$$
\widehat{\phi}(x, t)=\frac{1}{3} \sum_{i=-1}^{1} \phi(x+i a, t) .
$$

In what follows such a neighbourhood average of a quantity $X$ is represented by the hat symbol $\widehat{X}$. The preferred direction $U(x, t)$ is then determined by

$$
U(x, t)= \begin{cases}1, & \text { if } \widehat{\phi}(x, t)>0 \\ -1, & \text { if } \widehat{\phi}(x, t)<0 \\ 0, & \text { if } \widehat{\phi}(x, t)=0\end{cases}
$$

Thus in the model of [19] the preferred direction is given deterministically by the local momentum and the model thus includes alignment. Following a determination of $U, \eta$ is the probability that the velocity is not updated to this value (and instead to $-U$ ). This probability is independent of the dynamical variables.

In this paper we generalise the model of [19] at step (ii) above to include the effects of centring and separation as well as alignment - this will be discussed in section 2.4. We also generalise to consider the case where $U$ is itself a stochastic function of local variables: a boid may determine its preferred direction from the local variables as \pm 1 or 0 with some probabilities (as will be motivated below). However, since $U$ appears linearly in (1), averaging over its possible values amounts to replacing $U$ by its expectation value $\bar{U}$ in (10):

$$
W_{ \pm 1}=\frac{1}{2}\left[1 \pm G\left(x_{\mu}, t\right)\right]
$$

where

$$
G(x, t)=(1-2 \eta) \bar{U}
$$

Thus the important quantity is $\bar{U}(x, t)$, the expectation value of the preferred direction which should be a function of the local density and velocity, for example. The expectation value of the updated velocity is given by $G(x, t)$. 
In the next subsection we propose an explicit form for $\bar{U}$ and hence $G(x, t)$. First it is useful to review the form of (5). The quantity $\operatorname{sgin}(G)$ is the velocity to which a boid is updated in the absence of noise, whereas $|G|$ is the certainty with which this outcome is attained. The uncertainty in the outcome comes from two sources, the first is due to random errors, controlled by the parameter $\eta$, where the boid moves against its preferred direction $U$. The second is from the stochastic nature of $U$ itself, which as we argue in section 2.3 comes from the uncertainty with which a boid perceives the local flock properties and therefore determines its preferred direction.

\subsection{Alignment}

In many models [19, 10, 18, 11] it has been shown that alignment alone is sufficient to produce complicated behaviour recognisable as flocking. Alignment is effected in our dynamics through $G$. In this work we consider $G$ to be of the form

$$
G=(1-2 \eta) \frac{\tanh (\beta V(x, t))}{\tanh (\beta)}
$$

where

$$
V(x, t)=\frac{\widehat{\phi}(x, t)}{\widehat{\rho}(x, t)}
$$

is the neighbourhood average boid velocity. The parameter $\beta>0$ controls how nonlinear $G(x, t)$ is as the function of $V(x, t)$. In the limit where $\beta \rightarrow \infty$ the majority rule case (3) is in effect. In the limit $\beta \rightarrow 0$ we obtain a linear function. The form (6) addresses several deficiencies in (3). Firstly (6) is analytic, whereas (3) is non-analytic. Secondly with (6) boids become sensitive to increasing majority size, as might be expected in physical systems. In the following section we will see how this second intuitive feature can arise out of errors in local flock perception.

\subsection{Justification of form of $G$ via sampling argument}

We now turn to a justification of the form (6) from microscopic considerations. We present an argument amounting to a simple algorithm for the determination of $G$ based on sampling of neighbours. In the model of [19] boids determine $U$ with perfect knowledge of all neighbouring boids (and perfect ignorance of other boids); hence a local majority is determined with certainty. Consider instead the situation where a boid can only make $M$ observations of its neighbours, each observation is of one boid selected randomly from neighbouring boids with replacement. (Also note that from the definition of neighbourhood a boid acts as a neighbour to itself.) The Majority rule applied to the sample of $M$ boids then requires a fixed observation time $O(M)$ regardless of local density. This is in contrast to the strict majority rule algorithm which requires information about all neighbours (13), there being no restriction on the potential number of neighbours. 
Consider a sample of $M$ neighbouring boids from the group of $3 \widehat{\rho}(x, t)$ neighbours, containing $3 \widehat{n}_{1}(x, t)$ rightward and $3 \widehat{n}_{-1}(x, t)$ leftward moving boids. With binomial probability $P(k), k$ will be selected from the rightward and $M-k$ from the leftward moving groups:

$$
P(k)=\left(\begin{array}{c}
M \\
k
\end{array}\right)\left(\frac{\widehat{n}_{1}}{\widehat{\rho}}\right)^{k}\left(\frac{\widehat{n}_{-1}}{\widehat{\rho}}\right)^{M-k} .
$$

The preferred direction is determined from the sample as $U_{M}=\operatorname{sign}(2 k-M)$. The expected updated velocity $G_{M}$ is, taking $M$ is odd,

$$
G_{M}=(1-2 \eta) \bar{U}_{M}=(1-2 \eta) \sum_{k=0}^{\frac{M-1}{2}}[P(M-k)-P(k)] .
$$

Using the substitution

$$
\widehat{n}_{j}(x, t)=\widehat{\rho}(x, t) \frac{1+j V(x, t)}{2},
$$

$G_{M}$ can be expanded as an odd polynomial in $V$ :

$$
G_{M}=(1-2 \eta) \sum_{k=0}^{\frac{M-1}{2}} \sum_{i=0}^{k} \sum_{j=0}^{\frac{M-1}{2}-k} \frac{V}{2^{M-1}}\left(\begin{array}{c}
M \\
k
\end{array}\right)\left(\begin{array}{c}
k \\
i
\end{array}\right)\left(\begin{array}{c}
M-2 k \\
2 j+1
\end{array}\right)(-1)^{i} V^{2(i+j)} .
$$

A comparison of the two forms of $G$ (10) and (6) shows a qualitative correspondence between increasing $M$ and $\beta$. For example, we have from (10)

$$
\begin{aligned}
& G_{1}(x, t)=(1-2 \eta) V(x, t) \\
& G_{3}(x, t)=(1-2 \eta) \frac{V(x, t)}{2}\left(3-V(x, t)^{2}\right) \\
& G_{5}(x, t)=(1-2 \eta) \frac{V(x, t)}{8}\left(15-10 V(x, t)^{2}+3 V(x, t)^{4}\right),
\end{aligned}
$$

whereas expanding ([6) in powers of $V$ yields

$$
G(x, t)=(1-2 \eta) \frac{\beta V(x, t)}{\tanh (\beta)}\left(1-\frac{\beta^{2}}{3} V(x, t)^{2}+\frac{2 \beta^{4}}{15} V(x, t)^{4}+O\left(V(x, t)^{6}\right)\right) .
$$

In the two limiting cases $G_{M \rightarrow \infty}=\lim _{\beta \rightarrow \infty} G(x, t)$ and $G_{M=1}=\lim _{\beta \rightarrow 0} G(x, t)$ the correspondence is exact.

For intermediate values of $M$ it is possible to define an approximate correspondence through a function $\beta(M)$ using one of several schemes. Anticipating our mean field treatment below (section 4 ) we choose to match the gradient of $G$ with respect to $v$ at the origin:

$$
\beta(M)=\frac{1}{2^{M-1}} \sum_{k=0}^{\frac{M-1}{2}}\left(\begin{array}{c}
M \\
k
\end{array}\right)(M-2 k)=\frac{M}{2^{M-1}}\left(\begin{array}{c}
M-1 \\
\frac{M-1}{2}
\end{array}\right)
$$

This yields, for example, $\beta(M=3$ or 4$)=1.5$ and $\beta(M=25$ or 26$) \approx 4$.

To summarise we have argued that the form ([6), parametrised by $\beta$, for the expected updated velocity qualitatively corresponds to (but has a simpler form than) the result of taking a sample of $M$ neighbours. The correspondence $\beta(M)$ is quantified in (111). 


\subsection{Centring and Separation}

Centring, the tendency to move towards the local centroid $\omega(x, t)$, defined as

$$
\omega(x, t)=\frac{\sum_{i=-1}^{1} i \rho(x+i, t)}{\sum_{i=-1}^{1} \rho(x+i, t)},
$$

can be introduced through $G$ in an analogous way to alignment. If a relative importance of $\kappa$ is assigned to the effect of centring over alignment in any observation then (6) can be generalised to

$$
G(x, t)=(1-2 \eta) \frac{\tanh (\beta((1-\kappa) V(x, t)+\kappa \omega(x, t)))}{\tanh \beta} .
$$

The desire for separation, typically through hard core repulsion, is very restrictive in one dimension. If instead we consider separation as some cost associated with moving through a dense region then it amounts to a tendency to move away from the centroid (opposite to centring). In order to include these effects together we introduce a capacity, $C$, which is the capacity at which the relative strengths of these two effects neutralise. When the local density $\widehat{\rho}(x, t)$ is greater than $C$ the desire for separation exceeds the desire to centre, and vice versa. Thus, our most general form for $G$ is chosen as

$$
G(x, t)=\frac{(1-2 \eta)}{\tanh \beta} \tanh \left\{\beta\left[(1-\kappa) V(x, t)+\kappa \frac{C-\widehat{\rho}(x, t)}{C+\widehat{\rho}(x, t)} \omega(x, t)\right]\right\} .
$$

Note that (12) is the limit $C \rightarrow \infty$ of (13).

At this stage it is useful to summarise the parameters of the model with our choice of $G$ : the capacity $C$ is the density where the separation tendency surpasses the centring tendency; $\kappa$ determines the relative strengths of spatial density (centring and separation) and alignment effects. Thus $\kappa$ and $C$ determine the relative importance of Reynolds' three flocking criteria. The parameters $\eta$ and $\beta$ may both be considered as sources of noise acting through $G$ : $\beta$ introduces sensitivity to different field strengths while $\eta$ introduces uncorrelated errors. With reference to section 2.3 it is useful to consider the noise from $\beta$ as arising out of stochastically sampling the local flock to determine the boid's preferred direction whereas $\eta$ encompasses additional error sources once the preferred direction has been selected.

\section{Computer Simulations}

\subsection{Observed Regimes}

Numerical simulations were undertaken for a variety of noise levels $(\beta$ and $\eta)$, and flocking parameters $(\kappa$ and $C)$. Run time constraints limited our investigations to systems of size upto 1024 sites and times upto $10^{7}$ timesteps, where one timestep is sufficient time for each boid to be updated once on average ( $N$ updates). We found four characteristic and robust behaviours which we now discuss, alongside a myriad of intermediate behaviours. 


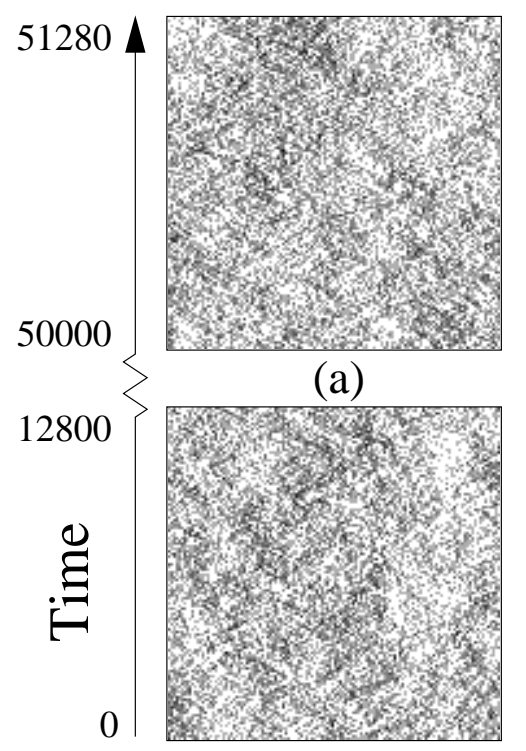

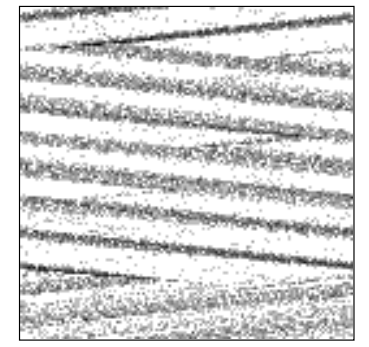

(b)

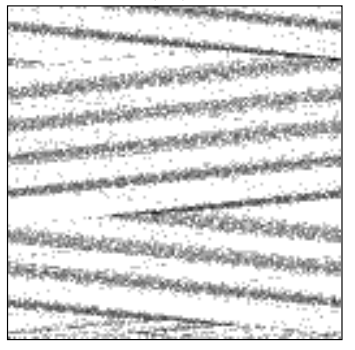

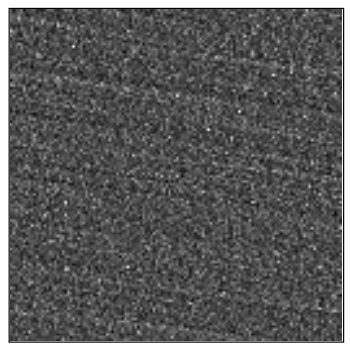

(c)

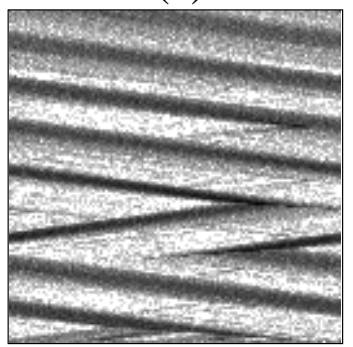

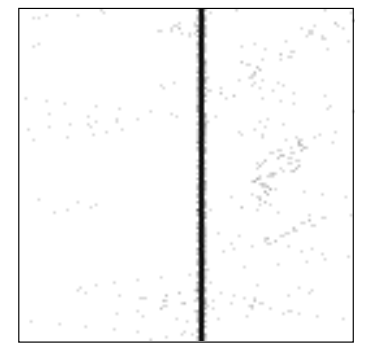

(d)

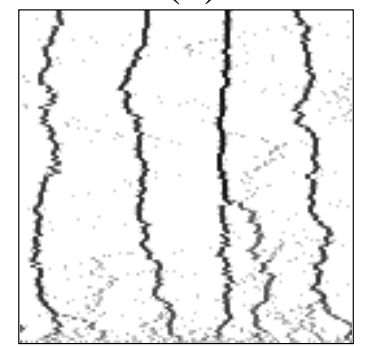

Figure 1: Figures show the development of systems in space time plots from random initial density and velocity distributions on systems with $L=128$ sites. The greyscale measures density $\rho(x, t)$ on a logarithmic scale. In this and following figures parameter sets are indicated [thus] (a) $[\eta=0.2, \beta=1, \kappa=0.5,\langle\rho\rangle=1, C \rightarrow \infty$ ] A disordered system showing no sustained correlations in density or velocity. (b) $[\eta=0.02, \beta=8, \kappa=0.5,\langle\rho\rangle=1$, $C \rightarrow \infty$ ] An alternating flock exhibiting a non-zero velocity between regular reversals. (c) $[\eta=0.125, \beta \rightarrow \infty, \kappa=0,\langle\rho\rangle=8]$ A homogeneous flock having homogeneous density and fixed global velocity. emerging from transient alternations. $(\mathrm{d})[\eta=0.02, \beta=8$, $\kappa=0.75,\langle\rho\rangle=1, C \rightarrow \infty]$ A dipole system consisting of several dipoles separated by low density homogeneous domains undergoes a slow coarsening process towards a single large dipole. (For the definition of a dipole see text.) 
The first characteristic behaviour (figure $1 \mathrm{i}$ ) is the disordered state. This state has homogeneous density and a global velocity of zero, global velocity being the mean velocity of a boid in the system

$$
\langle v\rangle=\frac{\langle\phi(t)\rangle}{\langle\rho\rangle} .
$$

This state persists at noisy parameter sets (high $\eta$, low $\beta$ ) especially where Reynolds' effects exist at equivalent strengths or where separation dominates $(\kappa \gtrsim 0.5$ and $C \lesssim\langle\rho\rangle)$.

The alternating flock is the second regime (figure 1b). This system undergoes a repeating pattern of cohesive traversals interspersed by rapid reversals, similar to that of [19].

The homogeneous flock as shown in figure 1 is a flocking state which complements the alternating flock. The homogeneous flock has a homogeneous density and fixed non-zero global velocity, $\langle v\rangle$. This regime is found to be unstable where noise is high or where global density is low, and is resilient only to small levels of centring or separation. The homogeneous flock is often established following an alternating transient as shown in the lower part of figure [1. The homogeneous flock can also be observed in the model of [19] but at larger densities, or smaller system sizes than those considered there

The final regime observed is a dipole state (figure 1 $\mathrm{d}$ ), which exists in systems where centring is the dominating Reynolds' effect. A dipole is a dense, localised structure. As we shall discuss in detail (in section 3.3) a dipole has a well defined centre with $G$ pointing inwards trapping boids, and a sharply decaying density profile at its edges. A system of one or many dipoles can exist on the array, either as direct neighbours or separated by depopulated domains. As can be seen in (figure 1d) a slow coarsening process is observed in a system of many dipoles, whereby small dipoles are eliminated and the boids are absorbed by larger dipoles. The dipole states exist for large values of $\kappa$ and $C$ i.e. relatively weak alignment and separation.

These four regimes all have readily characterised behaviours and occupy characteristic regions of parameter space as shown in figure 2 which describes the case $C \rightarrow \infty$ (absence of separation). Figure 2 may be thought of (and we shall refer to it) as a phase diagram. However, the different regimes are not truly "phases" as the boundaries between them depend on system size and, as we shall see below, the homogeneous flock regime actually disappears in the limit of large system size. In the following subsection we shall discuss in more detail the flocking and dipole regimes.

\section{2 $\quad$ Flocking regimes}

The alternating flock was originally studied in [19] where only alignment was present. Here we find that it persists in systems with all three Reynolds' effects present provided alignment dominates $(\kappa \lesssim 0.5)$ and $\beta$ remains finite. In its traversing stage a single dense group of boids (the flock), confined within a non-spanning section of the lattice, slowly flows across the system at a consistent non-zero velocity and diffuses. At a later time the reversal mechanism begins: this is initiated by a fluctuation near the front of the flock causing a local reversal in momentum. The fluctuation moves against the direction of the flock and grows in density and 


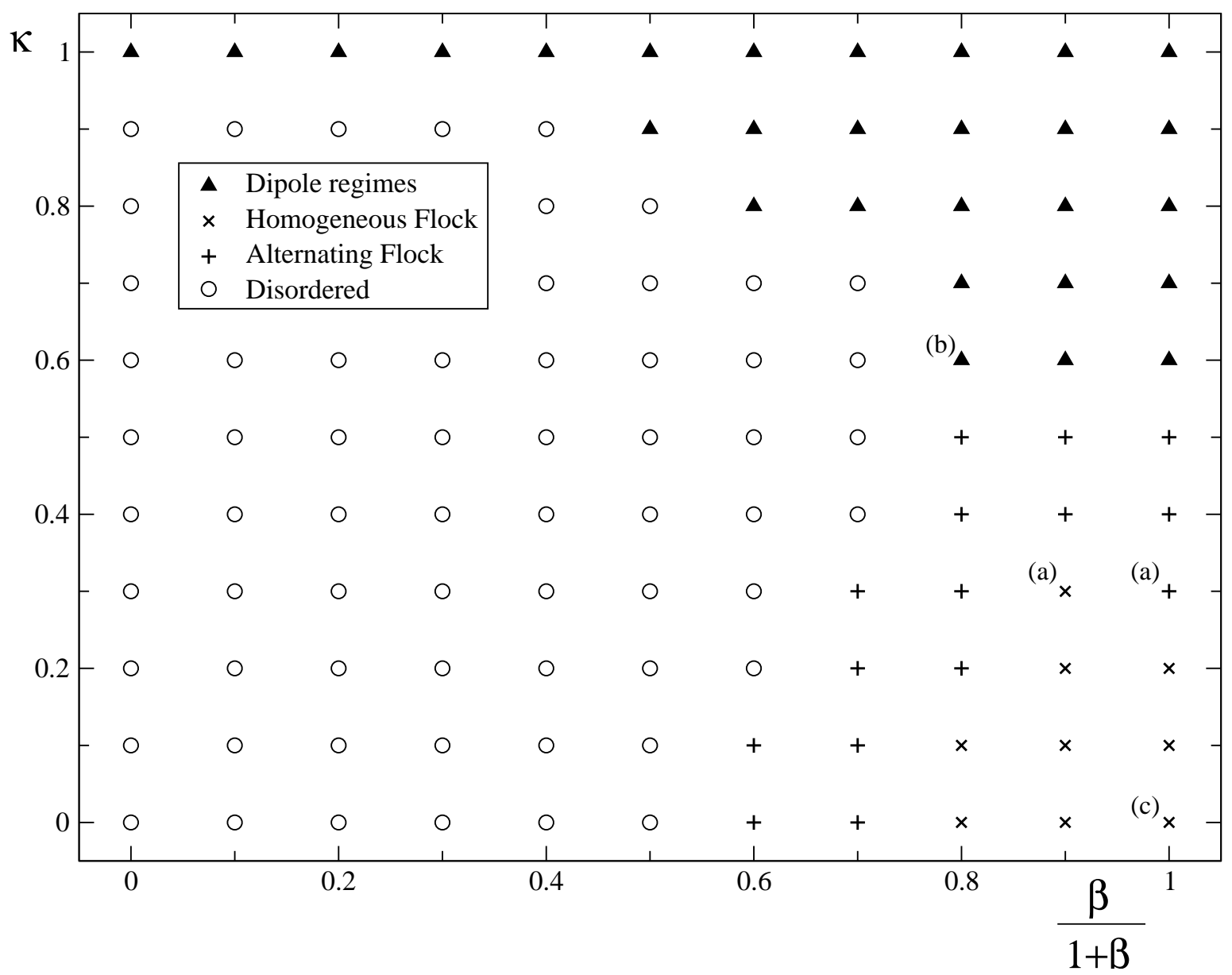

Figure 2: Diagram in the $\kappa-\beta /(1+\beta)$ plane showing the regimes present in a system with $L=128,\langle\rho\rangle=4, \eta=0.05$ with alignment and centring effects only $(C \rightarrow \infty)$. The dipoles, alternating flock and homogeneous flock all occupy characteristic regions. Intermediate states at the boundaries of these regions are characterised by mixed behaviours: (a) Flocks behave for sustained periods as both homogeneous flocks and alternating flocks, (b) dipoles begin to traverse large distances in single motions. The model of [19] is case (c), a homogeneous flock for this parameter set and system size. 
momentum until it passes entirely through the flock inverting its velocity. At this time the traversing behaviour of a flock with opposite velocity is established. Initiation of reversals can occur either internally or by collision with boids external to the flock. While not all large momentum fluctuations initiate a full reversal, they occur often enough to reverse the flock before it spreads out to occupy the whole lattice through diffusion. The reversals occur stochastically with no fixed period but there is a well defined mean time between reversals.

In the alternating flock instabilities occur at the front edge of the flock. In [19] it was established that the reversal timescale for alternating flocks is $O(\ln N)$ where $N$ is the number of boids. The argument is that this is the timescale on which the front edge becomes susceptible to relatively large momentum fluctuations, due to its low density. In our model there is a new form for $G$, but logarithmic timescales appear to persist for the alternating regime as shown in figure $3 \mathrm{~b}$.

As was shown in figure 1k, from many initial conditions a homogeneous flock develops after an initial transient alternating flock. However many homogeneous flocks are temporarily unstable towards an alternating flock profile even after a long time in the homogeneous regime. Such a process occurs for the homogeneous flock of figure 1p after 58000 timesteps. In the breakdown process a fluctuation in momentum, initially confined to a few sites, creates a disturbance which moves through the flock in a manner similar to the reversal mechanism in alternating flocks. Figures $4 a, b, c$ show the progression of the fluctuation through the flock to a point where global velocity is completely inverted. Before the flock returns to a homogeneous flocking regime there are several further reversals. The localisation of the boids and high reversal rate during this period characterise a transient alternating flock state (see figures $4 \mathrm{~d}, \mathrm{e})$.

It appears that the temporary failure of the homogeneous flock arises out of a large local fluctuation in momentum. During flock reversal the initial fluctuation acquires boids from the oncoming flock and continues to grow in momentum and stability. Although it appears that the size and type of disturbance required might vary for different systems, the rate at which such disturbances occur over the system should be proportional to the number of nucleation sites. Within the homogeneous flock, there is a homogeneous density so the number of nucleation sites is proportional to $L$, thus we expect the breakdown time for the flock, $T_{B}$ to behave as $1 / L$. In figure 3 a we take the mean time a homogeneous flock of positive momentum first reverses and attains a negative global velocity as the flock breakdown time. We fitted the data by $T_{B} \approx \frac{L}{4}+A L^{-B}$, where we expect $B$ to be approximately 1 . The constant $\frac{L}{4}$ is our approximation to the time for the fluctuation to pass through half the flock. Such a prediction appears to closely match computer simulations as illustrated in Figure [3a.

The previous paragraph implies that for large systems the homogeneous flock will always become unstable due to nucleation of momentum fluctuations. To quantify this, consider how the breakdown of the homogeneous flock to an alternating regime is initiated by a sequence of successive microscopic flips against the preferred direction sufficient to invert the momentum. The number of flips required for this to occur will be proportional to the local density, $\langle\rho\rangle$, and number of sites over which $G$ is determined (range, $r=3$ in our simulations). Given that a flip occurs with probability $P_{F}=\frac{1-|G|}{2}$, flock breakdown will be 

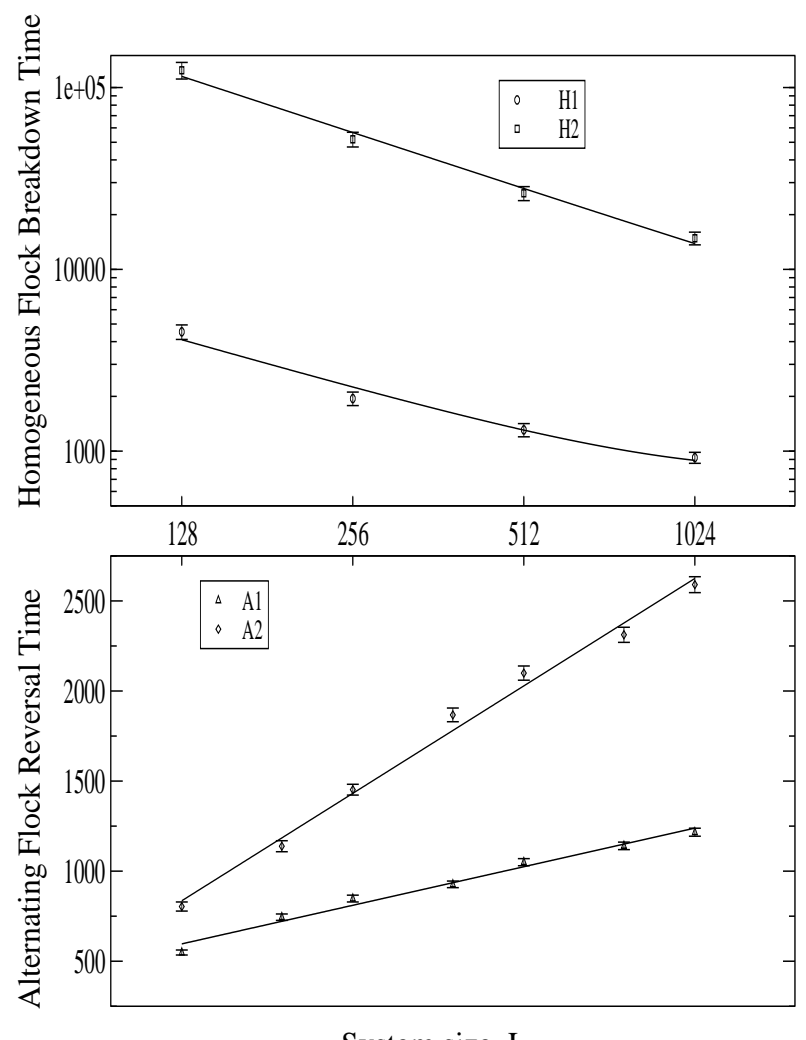

System size, L

Figure 3: a) Breakdown times for homogeneous flocks and b) reversal times for alternating flocks vs $\ln L$ where $L$ is the system size. Data is averaged over 100 and 1000 breakdowns and reversals respectively. (a) H1: $[\eta=0.0413, \beta=1, \kappa=0,\langle\rho\rangle=10], \mathrm{H} 2:[\eta=0.125, \beta=$ $\infty, \kappa=0,\langle\rho\rangle=8]$. The straightline is a least squares fit to $T=\frac{L}{4}+A L^{-B}$ (see text). The exponent is $B=1.03$ for $\mathrm{H} 2$, and 0.89 for H1. (b) A1: $[\eta=0.005, \beta=2, \kappa=0,\langle\rho\rangle=1]$, A2: $[\eta=0.02, \beta=8, \kappa=0.25,\langle\rho\rangle=1, C=\infty]$. The reversal times in alternating flocks are logarithmic and the straight lines are least squares fits to the data. 

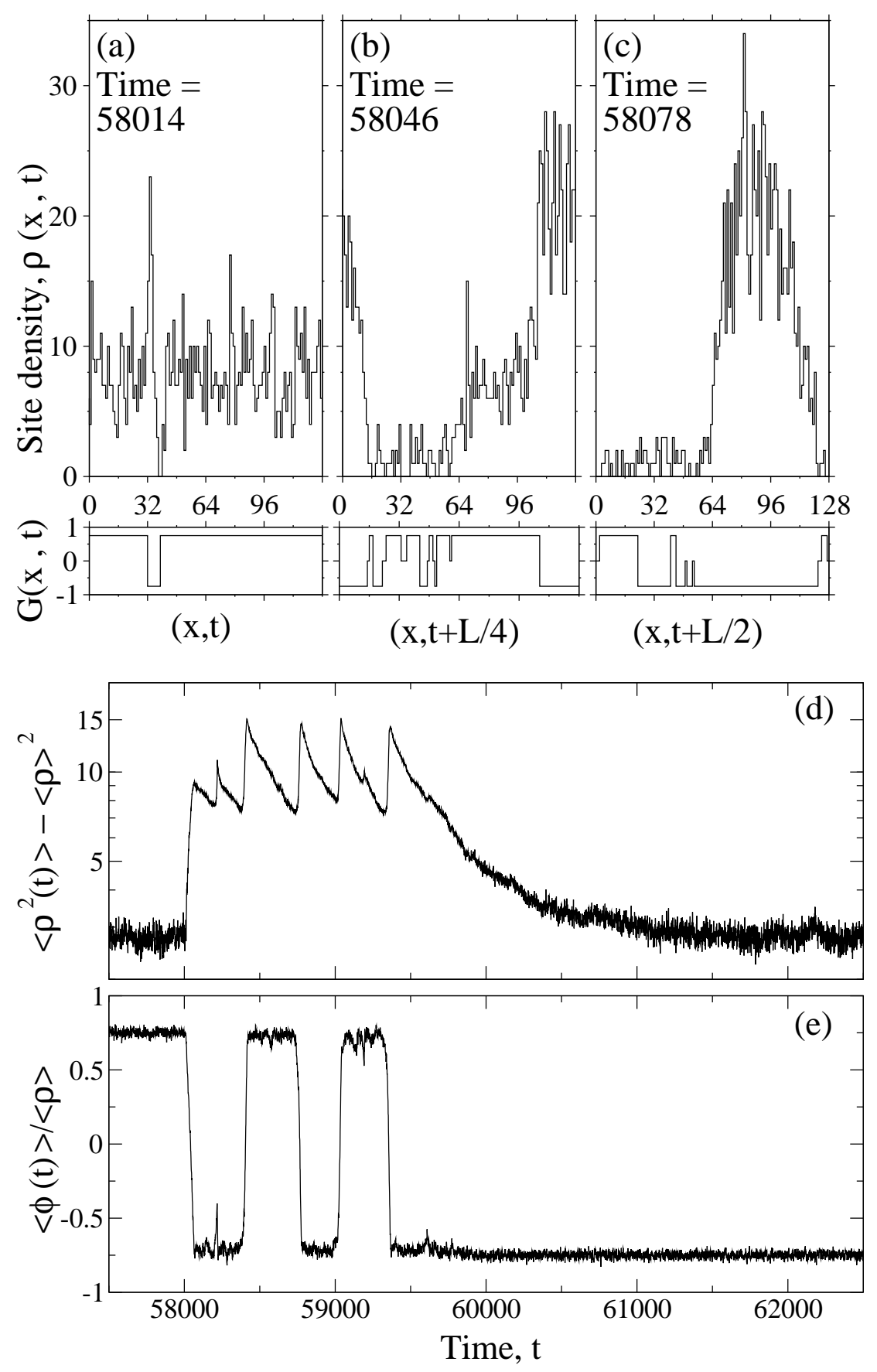

Figure 4: The Homogeneous flock of 1 undergoes a rapid change of behaviour. (a,b,c) Density and $G$ are plotted as a function of position at fixed times. a) $t=58014$ : values are consistent with a homogeneous flock throughout except near site 32 where a localised momentum fluctuation exists. (b) $t=58046$ : The interface between the two opposing domains created moves leftwards and the fluctuation gathers momentum. (c) $t=58078$ : Within a short space of time the entire flock is reversed, and the density and $G$ profiles are characteristic of an alternating flock. Following this event an alternating flock is temporarily established, before an homogeneous flock reemerges.

$(\mathrm{d}, \mathrm{e})$ A measure of the spatial distribution of boids and the global velocity plotted against time from before the breakdown, through the temporary alternating flock until the emergence of a homogeneous flock. 
initiated with probability $\sim L P_{F}^{k r\langle\rho\rangle}$ in any timestep. Therefore one expects the typical time $\tau$ for the flock to flip to be $\tau \sim L^{-1} P_{F}^{-k r\langle\rho\rangle}$. This implies that for the homogeneous flock to be stable over times $\tau \sim L^{\alpha}$ where $\alpha>0$, a density $\langle\rho\rangle \sim \ln L$ would be required. We conclude that for finite range and density no homogeneous flocks will be stable in the thermodynamic limit $(L \rightarrow \infty)$. However, as we have seen in Figure 2 on intermediate size systems there are well defined regions of parameter space where homogeneous flocks do exist.

Finally we examine the impact of varying the strength of centring and separation on flocking states. The alternating flock persists even when centring is a relatively strong effect, whereas the homogeneous flock typically becomes unstable towards an alternating flock under such conditions. Thus in the phase diagram in figure 2, for large enough $\beta$, increasing $\kappa$ leads to transitions from the homogeneous to the alternating flock and thence to the dipole state. The phase diagram with the inclusion of separation (finite $C$ ) is qualitatively similar. Perhaps surprisingly, decreasing $C$ does not substantially affect the alternating flock.

\subsection{Dipole Regimes}
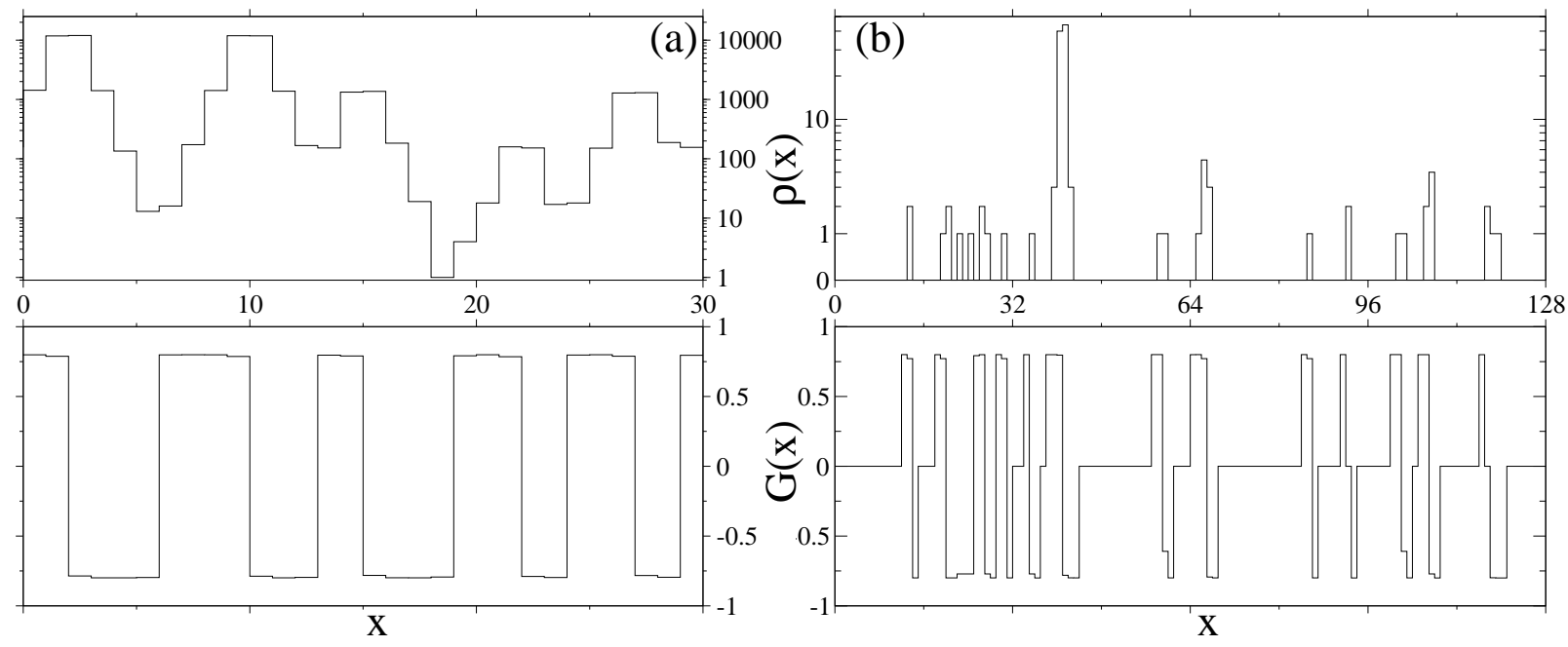

Figure 5: $[\eta=0.1, \beta=4, \kappa=0.75, C \rightarrow \infty]$. Density Profiles (upper panels) and profiles of the expected updated velocity, $G$, (lower panels) after 50000 timesteps. (a) At high density $\langle\rho\rangle=2000$ domains, with exponentially decaying densities and fixed velocity, bounded by discontinuities in $G$ are clear. (b) At density $\langle\rho\rangle=1$ dipoles occupy a small portion of the lattice, but contain the majority of boids. There are large portions devoid of boids with $G=0$.

The dipole regime is characterised by single or multiple localised structures. These may be stationary or slowly moving. The density is maximal at the centre of the dipole and the density profile is symmetric about the centre. However, to the left of the centre $G$ is positive but to the right of the centre $G$ is negative. Thus the structure traps particles in such a way that the net flux of boids across the centre of the dipole is zero. 
In figure 5 we illustrate two systems, one at high density and the other at densities typical of our other simulations (approximately 1). In the case of high density it can be seen that the system is dominated by adjacent large dipoles. Each of these dipoles has an exponentially decaying density to a boundary with another dipole, and a steady value for $G$ trapping boids. In this system there is slow redistribution of boids across boundaries towards larger dipoles. In the second system density is lower and the exponential density profiles are not obvious; instead there is one large dipole and several smaller dipoles. The dipoles span a very small fraction of space and are not in direct contact with others. The gaps between the dipoles are essentially homogeneous regions of very low density but small dipoles (of size 2-5 boids in the figure) regularly form and evaporate away. Occasionally a small dipole arising out of the low density region can grow to supersede larger dipoles.

In figure 6] details of a typical coarsening process, whereby small dipoles are eliminated and large dipoles grow, is illustrated. Figure [6] shows the development of a system, after an initial transient period when many dipoles form, through an intricate coarsening process. Figure 6a shows the system coarsening into two large dipoles and eventually (at a later time not included in [6a) one large dipole emerges. This may be seen from figures [6 b,c which plot the statistics of the sizes of the dipoles remaining in the system. The regions in between the dipoles is a low density domain containing independent boids which combine sporadically to form small transient dipoles. In the fully coarsened system there is a relatively small probability of a boid being elsewhere than the single dominant dipole - all dipoles of intermediate size disappear.

In the low density regime (as in 50) the exchange of boids between dipoles occurs through the homogeneous low density domains. Dipoles may lose boids through stochastic fluctuations at their boundaries. If two adjacent dipoles have such noise-induced boid loss at different rates, there will be a net flux across this domain towards the more stable dipole. Numerically we have observed that in fact smaller dipoles have a higher loss rate than larger ones. Therefore the rate of boid loss depends on the fine details of the dipole profile which depend on the dipole size. (An assumption of a pure exponential density profile for all dipoles would imply no dependence of the rate of boid loss on dipole size.) Other effects might also be important, for example the greater mobility of smaller dipoles.

Dipoles are created and sustained where centring is the dominant effect. Within dipole systems increasing alignment (decreasing $\kappa$ ) is most noticable in the resulting greater mobility or wandering 21] of dipoles (and independent boids); a significant process since it hastens the coarsening of the system, especially in the initial stages. Wandering can occur in any size dipole but is most prominent in smaller dipoles. Typically wandering is initiated by a net flux of boids across the centre of the dipole causing the centre to shift. This motion can be induced or enhanced by alignment, since the matching of velocities in such a process favours the collective motion. With alignment sufficiently strong, this process can be selfreinforcing to the extent that a sequence of movements or sustained translations (flocking) become possible.

Dipole systems are characterised by their central region of high density. However, we might expect such a profile to be suppressed by separation. The inclusion of separation in 


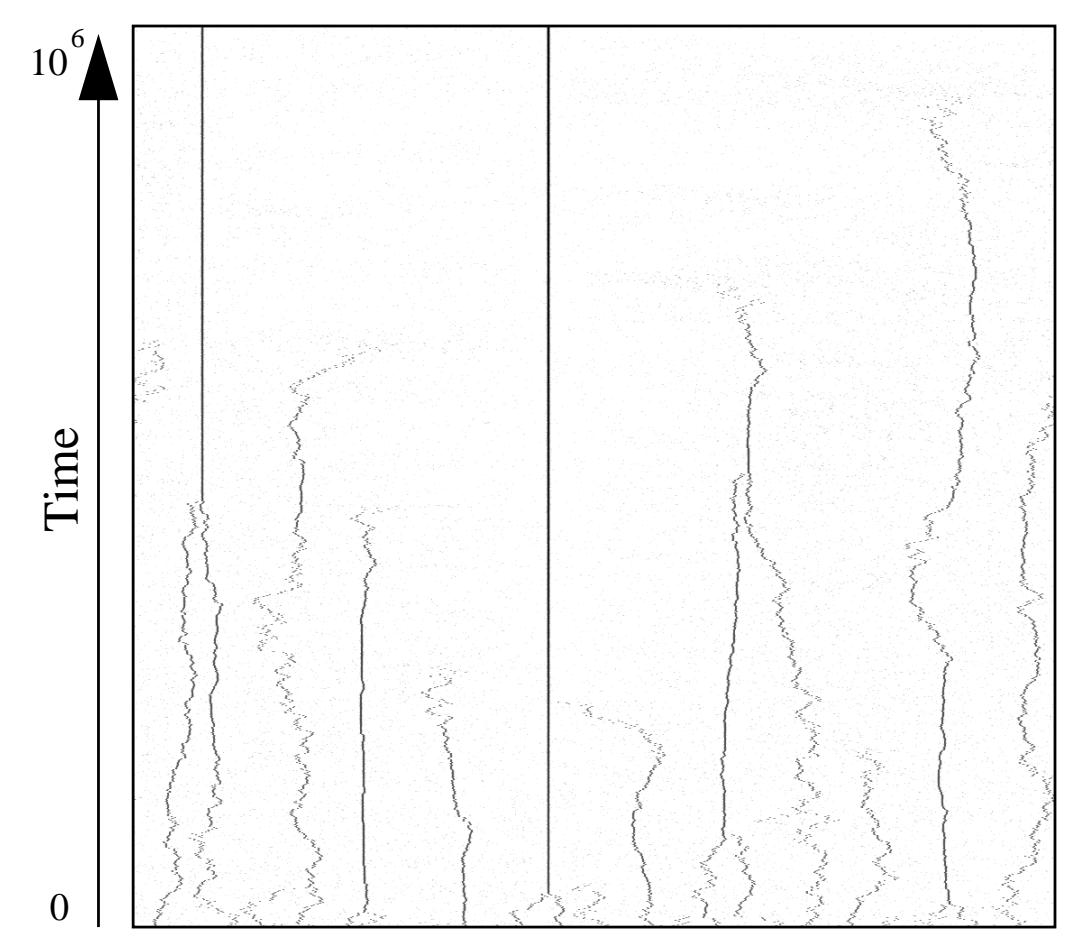

(a)

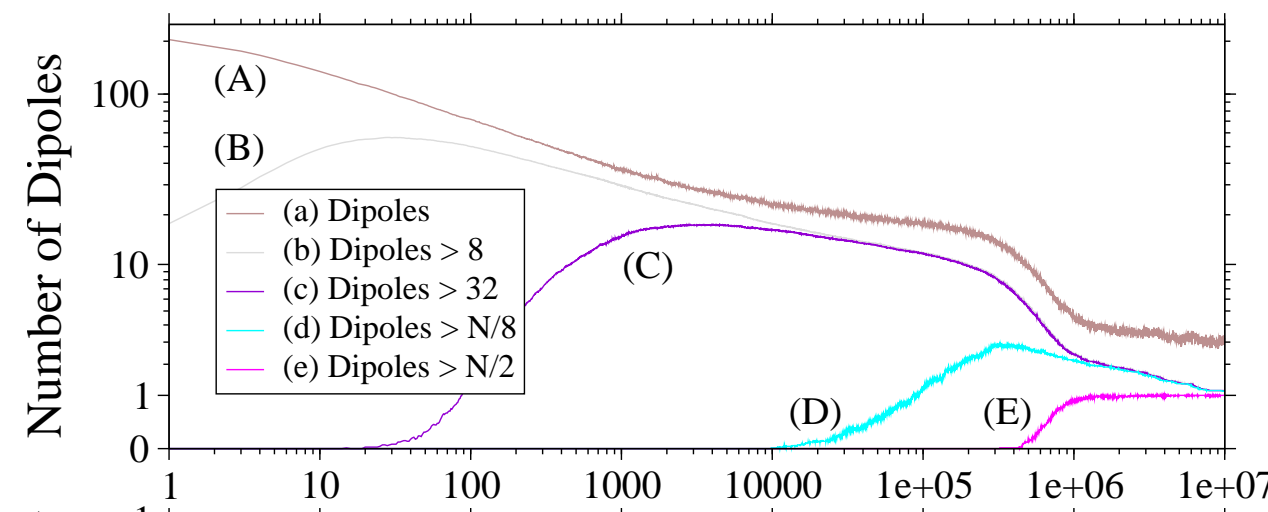

(b)

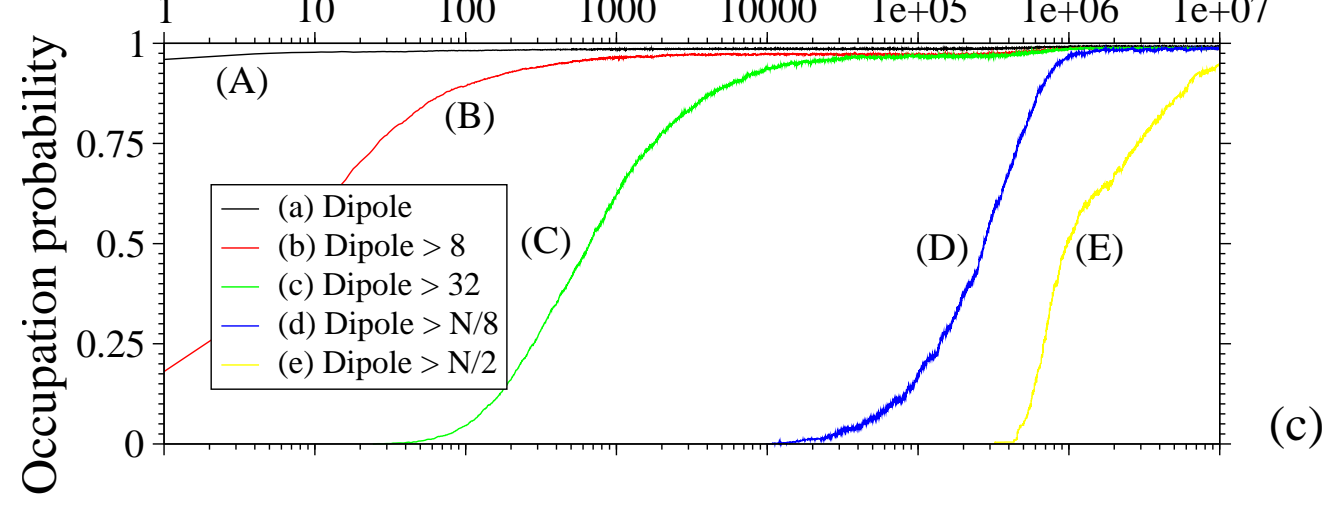

Figure 6: System with same parameter set as figure $1 \mathrm{~d}$ on a larger system of $L=1024$. (a) A typical space-time plot starting from random initial conditions. After an initial transient period when the dipoles form, an intricate coarsening process takes place wherein small dipole eventually dissolve and large dipoles grow. (b,c) Statistical data on the remaining dipoles as a function of time averaged from 100 runs similar to (a). (b) The number of dipoles remaining of size greater than the values in $\$ 5$ cated in the legend: Eventually the number of dipoles of intermediate size decreases to zero (curves B,C,D,E converge to 1); After $10^{7}$ timesteps a single large dipole emerges alongside approximately 2 small transient dipoles. (c) The probability that a boid is contained within a dipole greater than the sizes indicated in the legend as a function of time: Only a small number of boids ever exist outside dipoles (A) and after sufficient time the maiority of boids are contained in a sinole laroe dinole (E). 


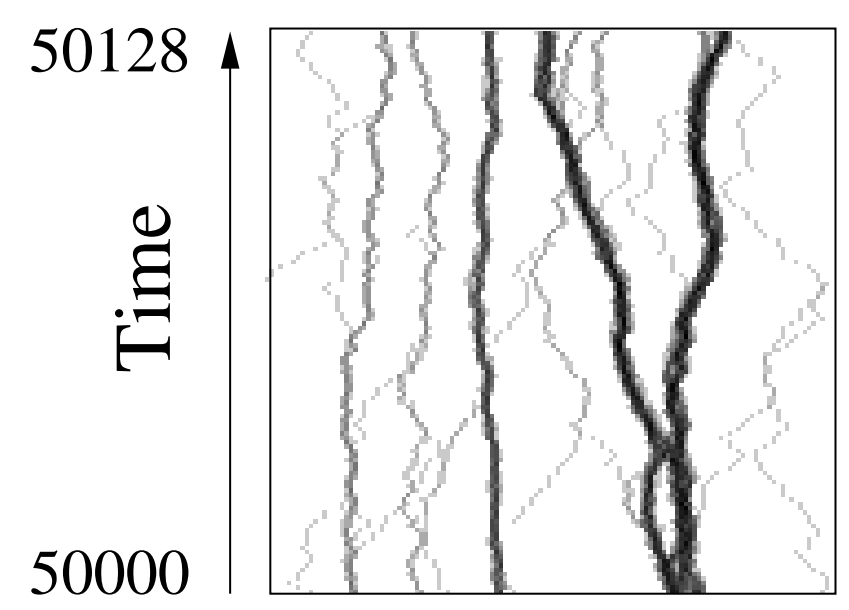

Figure 7: $\quad[\eta=0.02, \beta=8, \kappa=0.85,\langle\rho\rangle=1, C=20]$ This system contains two large fixed-density dipoles alongside small dipoles and solitary boids. In small dipoles centring is the dominant effect and wandering is limited, however in the two fixed-density dipoles large translations are observed. See text for explanation of the term fixed-density dipole.

dipole systems leads to a dramatic alteration of behaviour: where alignment is a small effect, and $\langle\rho\rangle<C<\infty$, a system of fixed-density dipoles can be observed. Fixed-density dipoles have reduced density at the centre, but the density is constant within an extended central region and only near the boundaries does one see the sharply decaying density profile, thus the density profile has a flat top. Within fixed-density dipoles alignment plays a much more important role than in comparably sized dipoles in the absence of separation, since at the centre of fixed-density dipoles the separation and centring tendencies tend to cancel allowing alignment to dominate. The relative stability of large and small fixed-density dipoles is left as an open question. Fixed-density dipoles can, for example, split from their centre and wander more substantially. These processes are appreciable in figure 7 where the mobility of the large fixed-density dipoles is second only to that of solitary boids.

\section{Mean Field Theory}

In order to understand some of the regimes observed numerically above, we now develop, from a mean field treatment of the full dynamics, a continuum model in which the density $\rho$ and neighbourhood velocity $v$ are continuous functions. In so doing we are able to identify a linearly stable flocking solution comparable to the homogeneous flock and a piecewise continuous solution comparable to the dipole regime. Furthermore we show that these solutions exist in numerical iterations of the mean-field equations. 


\subsection{Equations}

The equations satisfied by the mean-field density, $\rho$, and mean-field neighbourhood velocity, $v$, are derived in appendix $\mathrm{A}$ and read

$$
\begin{aligned}
& \frac{\partial \rho}{\partial t}=-\frac{\partial}{\partial x}(\rho G)+\frac{1}{2} \frac{\partial^{2}}{\partial x^{2}} \rho \\
& \frac{\partial v}{\partial t}=-v+G+\frac{1}{\rho}\left[v \frac{\partial}{\partial x}(\rho G)-\frac{\partial}{\partial x} \rho\right]+\frac{1}{2 \rho}\left[-v \frac{\partial^{2}}{\partial x^{2}} \rho+\frac{\partial^{2}}{\partial x^{2}}(\rho G)\right]
\end{aligned}
$$

where

$$
G(v, \omega)=\frac{1-2 \eta}{\tanh \beta} \tanh [\beta((1-\kappa) v+\kappa \omega)]
$$

and

$$
\omega=\frac{2}{3 \rho} \frac{\partial}{\partial x} \rho
$$

Equation (15) is rather easy to understand: note that, since $G$ is the mean velocity of boids, $\rho G$ is a mass current, thus the equation is a continuity equation with a current and a diffusive term. The equation for the neighbourhood velocity $v$ (16), on the other hand is more complicated.

\subsection{Steady State Solutions and Linear Stability}

We now look for steady state solutions of the above equations, for which we set the lhs of (1516) to zero. Further, we look for homogeneous solutions where spatial derivatives are zero. The global density may be freely chosen, in these solutions $\rho(x, t)=\langle\rho\rangle$, whereas velocities are determined by (16) as $v=G(v, 0)$ which yields

$$
v=(1-2 \eta) \frac{\tanh (\beta(1-\kappa) v)}{\tanh \beta} .
$$

Thus, there are either one or three solutions to the mean field theory which are homogeneous in density.

For all parameter sets one solution to this is $v=0$, the disordered solution, whilst for certain values of $\beta, \kappa$ and $\eta$ two flocking solutions $v=v_{ \pm}$exist, corresponding to the homogeneous flock. The existence of the flocking solutions is determined by the gradient in $G$ with respect to $v$ at the origin. Since $G$ is a concave function of $|v|$ a flocking solution of (19) requires that $G^{\prime}(0,0) \geq 1$. Thus the existence of this flocking solution is dependent on the following criterion being satisfied:

$$
\frac{\tanh \beta}{\beta} \leq(1-2 \eta)(1-\kappa)
$$

This is possible in systems with low $\eta$, but strong alignment (so that $\beta(1-\kappa)$ is large). 


\subsection{Piecewise Dipole Solution}

We show in this section how it is possible to determine a further steady-state solution of (1516) where the density is an exponentially increasing or decreasing function of $x$ and $v$ is a positive or negative constant respectively. Domains of these solutions may be pieced together so that the density is continuous to form dipole structures.

First, we assume a fixed value of $\omega$ implying by definition (18) a density profile

$$
\rho(x, t)=A \exp \left(\frac{3 \omega x}{2}\right)
$$

where $A$ is a constant. Next we propose a solution whereby within any domain $G$ and hence velocity are fixed. Then, from (15) we have

$$
0=-G(w, v) \frac{3}{2} \omega \rho(x)+\frac{9}{8} \omega^{2} \rho(x)
$$

which implies

$$
G(\omega, v)=\frac{3 \omega}{4} .
$$

The velocity $v$ must similarly be steady and (16) implies

$$
0=-v+G(\omega, v)+\frac{3}{2} \omega G(\omega, v) v-\frac{3}{2} \omega-\frac{9}{8} \omega^{2} v+\frac{9}{8} \omega^{2} G(\omega, v)
$$

which after substitution of $G$ from (23) simplifies to

$$
v=\frac{3 \omega}{4}\left[2\left(\frac{3 \omega}{4}\right)^{2}-1\right] .
$$

The condition required for a non-homogeneous solution is the consistency of (23) and (17), eliminating $G$ we obtain

$$
\omega=\frac{4(1-2 \eta)}{3 \tanh \beta} \tanh \left\{\beta\left[\left(\frac{7 \kappa}{3}-1\right) \frac{3 \omega}{4}+2(1-\kappa)\left(\frac{3 \omega}{4}\right)^{3}\right]\right\}
$$

Alongside the homogeneous density solutions, $\omega=0$, there are potentially dipole solutions of (25) $\omega_{ \pm} \neq 0$. Correspondingly $v$ takes values $v\left(\omega_{ \pm}\right)$via (24). One of these has rightward $G$ and exponentially increasing density, the other a leftward $G$ and exponentially decreasing density. A dipole is a localised structure consisting of a left hand region of exponentially increasing density and a right hand region of decreasing density. To satisfy periodic boundary conditions it is necessary that the system consists of complementary domains. Consider a boundary between the dipole domains: the two exponentially decaying or exponentially growing domains meet with equal density at such a point. The density gradient and other qualities are locally antisymmetric about the boundary; hence the net flux of boids will be zero and the boundary stationary (this would not be true for a dipole domain in contact with 
a homogeneous domain). Within each domain the presence of a boundary is not felt, and so domains of differing width and height can neighbour one another. Hence non-symmetric dipoles of differing sizes can be neighbours. Our solutions are therefore the set of coupled non-overlapping dipole systems (domain pairs $i$ ), each defined uniquely by the set of dipole centre positions, heights and widths.

These solutions are comparable to the full stochastic system at very high density (see figure 5a). Note that the larger dipoles in the sparser system (figure 5b) also approximate a pair of domains with the same fixed $G$ and $\omega$.

In numerical iterations of the mean field equations (see next subsection) the dipole structures were rounded at the cusps which form at domain boundaries. One expects this rounding and the physics at the cusps to be described by higher order terms in the lattice spacing $a$ which have been ignored (see Appendix A).

\subsection{Numerical Analysis}

Numerical iteration of the dynamical mean field equations (1516) allows further analysis of these states and comparison to full system dynamics. The iteration was carried out by rediscretising (1516) onto a lattice. We found the flocking $\left(\omega=0, v_{ \pm}\right)$, non-flocking $(\omega=$ $0, v=0)$ and dipole solutions $\left(\omega_{ \pm}, v\left(\omega_{ \pm}\right)\right)$to be present, in addition to systems comparable to the fixed-density dipole. The alternating flock however was never observed, nor were systems comparable to sparser dipole regimes.

Where a flocking solution is predicted, and not made unstable by the presence of centring (see section 4.6) a homogeneous flock with the anticipated global velocity (19) was always observed to emerge from any initial conditions.

In dipole regimes we found that the system iterated to a dipole system consisting of many dipoles. However the mean field dipole system does not exhibit a further coarsening process comparable to the full stochastic model. This is likely due to the absence of noise driven fluctuations which drive the coarsening process. On the other hand, where the initial boundary between domains does not fall perfectly on a lattice space boundary or centre a very slow redistribution of boids does occur in the mean-field system, but apparently at only an exponentially slow rate. This redistribution was not observed to cause the disappearance of any dipoles, nor did it appear to favour transfer to larger dipoles (is the case in the full stochastic system).

\subsection{Linear Stability of Mean Field Solutions}

We have developed a linear stability analysis of the disordered and flocking solutions to the mean field equations. This is done by linearising (15, 16), computing the evolution of the Fourier components then checking whether positive (unstable) eigenvalues exist. As the details are rather involved we do not present them here but quote some results. In the case $\kappa=0$, when the flocking solutions exist they are linearly stable and the disordered solution is unstable; when the flocking solution does not exist the disordered solution is stable. However, for sufficiently large $\kappa$ an instability will occur in both flocking and disordered solutions. 
This instability is expected to be with respect to stable dipole solutions, although we did not explicitly check the stability of dipole solutions.

Thus the equality in (20) defines a mean-field phase boundary between the disordered and possible flocking states. Comparing to Figure 2 this curve is in approximate agreement to the boundary between the disordered and alternating flock regimes. However for other parameter sets the boundary is less accurately predicted. Generally the boundary becomes increasingly more accurate as the density increases.

As well as the disordered/flocking phase boundary, the instability of the flocking solutions at large $\kappa$ is also consistent with Figure 2 where for large $\kappa$ dipole solutions appear. The only discrepancy remains the absence of alternating flocking solutions within the mean field theory: in regions where one sees an alternating flock in the full stochastic system the mean field theory has a homogeneous flock solution.

\subsection{Absence of Alternating Flock and Stability of Mean Field So- lutions}

As noted above the alternating flock was not expressed within the mean field theory, even in the time dependent form (15, 16). As we shall now discuss, the mean field theory is not capable of describing the alternating flock which is driven by fluctuations. However the flocking solution of the mean field theory does appear to correctly predict the velocity of the alternating flock between reversals i.e. the solutions of (19) proves to be in good agreement with transient results from the full stochastic system. We also note that we found numerically that the mean field theory correctly describes the evolution of the profile of an alternating flock as it spreads between reversals. However, whereas an alternating flock in the full stochastic system will inevitably reverse due to some fluctuation in momentum (as described section (3.2) the mean-field profile will keep evolving until the homogeneous flock is attained. Similarly, due to the absence of fluctuations the homogeneous flock will not reverse for any system size.

Clearly, to reproduce the alternating flock, a noise term should be added to the mean field equations (15) [16) thus turning them into Langevin equations. This would be done, following the proposal of [16, 18, by adding a noise term to the velocity equation (16). The equations (15, 16) would then be similar to those of [16, 18, although our velocity equation, which we derived from the microscopic dynamics, is rather more complicated and contains additional terms.

\section{Discussion}

In this work we have extended the flocking model of [19] to include all three of Reynolds' three effects - alignment, centring and separation - implemented in combination or independently. Within this model we demonstrated the robustness of the alternating flock highlighted in [19] to the addition of other Reynolds' effects. Also we showed the existence of two new regimes: the homogeneous flock and dipole structures. These are consistent with results 
from a mean field treatment which, in particular, correctly predicts the form of the dipole solutions. Furthermore, we investigated the coarsening process in the low density dipole regime.

We also derived from microscopic considerations the proposed form for the function $G$ (6). The algorithm presented in section 2.3 is not only intuitive but significantly is a fixed time algorithm, by which it is meant that the algorithm would involve sampling a fixed number of neighbouring boids whatever the density. Such algorithms are aspired to in flocking applications [3, 14] and are presumed to underly natural boid decision making [7]. Several ways to extend the algorithm could be to include a density, velocity or historical dependence in determining the sample, anisotropic spatial sampling or considering a direction selection rule other than majority. For example boids could require unanimity [20] amongst their observed neighbours to believe that any orientation is a worthy choice.

Reynolds' three effects were defined as required behaviours for simulating real flocks. In this paper we have shown several states which may characterise real systems. Firstly the homogeneous flock, which has many analogies in two or three dimensions, can be realised in some systems approximating one dimension: for example skaters confined to the outside of a rink, or fish in a ring shaped tank (as in the Boston Aquarium). The fixed density dipole is characterised by sharp edges and fixed internal density, and the ability to wander - either slowly, or with the inclusion of alignment, quickly and with a sustained orientation. These qualities, as well as as the ability to split from within, could describe a number of biological systems. Finally consider the reversal mechanism present in the alternating flock; such sharp reversals of direction are characteristic of the manoeuvres seen in many flocks, though they are poorly described by many models.

The numerical results obtained for the full stochastic system have some noteworthy similarities with previous studies. A flocking state is observed in the one dimensional continuous space model of [18] which appears to resemble an alternating flock. Dipole-like regimes have been observed in [21, where dense states are supported by a centring interaction. As with our dipoles the density at the centre of these systems grows rapidly with boid number. This effect is suppressed by a hard core repulsion, producing states similar to our fixed density dipoles. In 21] two dimensional systems also display wandering and oscillating (circling) behaviour. In [14] a two dimensional cellular automaton demonstrates a density-dependent state with symmetry-broken velocity. This density dependence arises from a hard capacity being placed on the number of boids which can occupy a single site; in effect the flocking state is destroyed by separation at high density.

Our mean field treatment has similarities with the continuum model in [18. Not only are the same states demonstrated, but the terms within the equations are comparable also. Special note is made in [16] of a term of the form $\frac{1}{\rho} \frac{\partial}{\partial x} G \frac{\partial}{\partial x} \rho$ which is present in our equation (16). This term relates to domain competition, and aids in an understanding of the reversal mechanism and breakdown of the homogeneous flock. As (16) describes very well the shape of a spreading flock, and it is believed that this shape allows certain fluctuations to cause the flock reversal mechanism [19, it might be hoped that the addition of noise into (1516) would allow the alternating flock to be further analysed. The coarsening process in low and high 
density dipole regimes may also be observed by allowing fluctuations to enter the dynamics at the boundaries of the dipoles.

Finally we mention one further variation of the model of [19] that we have studied [20]. To imitate the effects of inertia we modified step (iii) of our dynamics such that boids which decide to change direction must proceed through a temporary zero velocity state. Numerical simulations and a mean field treatment (which we do not present here) indicate that under such a scheme flocking solutions become more prominent and have higher global velocities. Although the flipping mechanism proceeds in much the same manner as the two velocity

case, the rate of reversal for both the alternating and homogeneous flock is reduced This is not surprising since more unfavoured flips are required to create momentum fluctuation leading to a sustainable reversal of the velocity.

Acknowledgements We thank Mike Cates and Jamie Wood for carefully reading the manuscript and for helpful discussions. The work was supported in part by EPSRC Programme grant GR/S10377/01.

\section{References}

[1] E. Bonabeau, L. Dagorn, P. Freon J. Phys. A: Math. Gen 31 L731-L736 (1998)

[2] P. D. Lorch, D. T. Gwynne Naturwissenschaffen 87 370-372 (2000)

[3] C. W. Reynolds, Computer Graphics 214 (1987)

[4] A. Jadbabaie, J. Lin, and A. S. Morse IEEE Transactions on automatic control 48988 (2003)

[5] R. Olfati-Saber, R.M. Murray IEEE Transactions on automatic control 491520 (2004)

[6] Y. Liu, K. K. M. Passino, M. Polycarpou IEEE Transactions on automatic control 48 $1848(2003)$

[7] S. Hubbard, P. Bank, S. Sigurdsson and K. G. Magnussen Ecological Modelling 174 359-374 (2004)

[8] W. J. Crowther, Proc. Instn. Mech. Engrs Part G (Journal of Aerospace Engineering) 218 111-124 (2004)

[9] J. Toner, Y. Tu and S. Ramaswamy Annals of Physics 318170 (2005)

[10] T. Vicsek, A. Czirok, E. Ben-Jacob, I. Cohen, and O. Shochet, Phys. Rev. Lett. 751226 (1995)

[11] J. Toner and Y. Tu, Phys. Rev. Lett. 754326 (1998)

[12] J. Toner and Y. Tu, Phys. Rev. E58 4828 (1998) 
[13] N. Shimoyama, K. Sugawara, T. Mizuguchi, Y. Hayakawa, M. Sano Phys. Rev. Lett. 7620 (1996)

[14] H. J. Bussemaker, A. Deutsch, and E. Geigant Phys. Rev. Lett. 7826 (1997)

[15] M. Aldana and C. Huepe, J. Stat. Phys. 112135 (2003)

[16] T. Vicsek, A. Czirok, I. J. Farkas and D. Helbing, Physica A 274 182-189 (1999)

[17] M. R. Evans Braz. J. Phys. 3042 (2000)

[18] A. Czirok, A. Barabasi, T. Vicsek, Phys. Rev. Lett. 82209 (1999)

[19] O. J. O'Loan and M. R. Evans, J. Phys. A. 32 L99 (1999)

[20] J. R. Raymond (Masters Thesis, University of Edinburgh, unpublished),

[21] H. Levine, W J. Rappel and I. Cohen Phys. Rev. E63 017101 (2001)

\section{A Derivation of Mean Field Equations}

In our mean field theory we shall approximate the density $\rho(x, t)$ (the number of particles at site $x$ and time $t$ ) and the momentum $\phi(x, t)$ (the number of right going minus the number of left moving particles at site $x$ and time $t$ ) by continuous functions and derive the evolution of these funcions by ignoring certain correlations. We begin with the dynamics of site $x$ in a single update, as defined by $\rho(x, t)$ and $\phi(x, t)$. Consider $\rho(x, t)$, in a single update this can increase if a boid selected from a neighbouring site moves into site $x$, or can decrease if a boid is selected from site $x$, with an associated probability:

$$
\begin{aligned}
& \rho(x, t+\delta t)= \\
& \begin{cases}\rho(x, t)+1, & \text { Probability }=\frac{\rho(x-a, t)}{N} W_{+}(x-a, t)+\frac{\rho(x+a, t)}{N} W_{-}(x+a, t) \\
\rho(x, t)-1, & \text { Probability }=\frac{\rho(x, t)}{N} \\
\rho(x, t), & \text { otherwise. }\end{cases}
\end{aligned}
$$

We then average over the events occurring between time $t$ and $t+\delta t$

$$
\rho(x, t+\delta t)=\sum_{\rho(x, t+\delta t)} \rho(x, t+\delta t) P_{\rho(x, t+\delta t)}
$$

and make the mean field approximation of factorising all averages.

Further we assume $\rho(x, t)$ and $\phi(x, t)$ vary smoothly on the time and length scales effective in a single update. With these assumptions we expand about site $x$ to second order (in lattice 
spacing $a$ ) and about $t$ to first order $\left(\frac{1}{N}\right)$.

$$
\begin{aligned}
\frac{\partial \rho}{\partial t} & =\left\{\rho-a \frac{\partial \rho}{\partial x}+\frac{a^{2}}{2} \frac{\partial^{2} \rho}{\partial x^{2}}\right\}\left\{W_{+}-a \frac{\partial W_{+}}{\partial x}+\frac{a^{2}}{2} \frac{\partial^{2} W_{+}}{\partial x^{2}}\right\} \\
& +\left\{\rho+a \frac{\partial \rho}{\partial x}+\frac{a^{2}}{2} \frac{\partial^{2} \rho}{\partial x^{2}}\right\}\left\{W_{-}+a \frac{\partial W_{-}}{\partial x}+\frac{a^{2}}{2} \frac{\partial^{2} W_{-}}{\partial x^{2}}\right\} \\
& -\rho .
\end{aligned}
$$

By introducing $G$ (5) the following equation can be determined. In this equation there is a diffusion term arising from the choice of random stochastic updates, and a current term controlled by the mass current $\rho G$.

$$
\frac{\partial \rho}{\partial t}=-a \frac{\partial}{\partial x}(\rho G)+\frac{a^{2}}{2} \frac{\partial^{2}}{\partial x^{2}} \rho .
$$

In a similar way one can develop an equation for $\phi(x, t)$ beginning with the full dynamics at $x, t$,

$$
\phi(x, t+\delta t)= \begin{cases}\phi(x, t)+1, & \text { Probability }=\frac{\rho(x-a, t)}{N} W_{+}(x-a, t)+\frac{n_{-1}(x, t)}{N} \\ \phi(x, t)-1, & \text { Probability }=\frac{\rho(x+a, t)}{N} W_{-}(x+a, t)+\frac{n_{1}(x, t)}{N} \\ \phi(x, t), & \text { otherwise. }\end{cases}
$$

Taking the expectation value and expanding to second order in $a$ and first order in $t$ :

$$
\begin{aligned}
\frac{\partial \phi}{\partial t} & =\left\{\rho-a \frac{\partial \rho}{\partial x}+\frac{a^{2}}{2} \frac{\partial^{2} \rho}{\partial x^{2}}\right\}\left\{W_{+}-a \frac{\partial W_{+}}{\partial x}+\frac{a^{2}}{2} \frac{\partial^{2} W_{+}}{\partial x^{2}}\right\} \\
& -\left\{\rho+a \frac{\partial \rho}{\partial x}+\frac{a^{2}}{2} \frac{\partial^{2} \rho}{\partial x^{2}}\right\}\left\{W_{-}+a \frac{\partial W_{-}}{\partial x}+\frac{a^{2}}{2} \frac{\partial^{2} W_{-}}{\partial x^{2}}\right\} \\
& -\phi
\end{aligned}
$$

Using (5) this can be simplified to its final form

$$
\frac{\partial \phi}{\partial t}=-\phi+\rho G-a \frac{\partial}{\partial x} \rho+\frac{a^{2}}{2} \frac{\partial^{2}}{\partial x^{2}}(\rho G) .
$$

Defining the (neighbourhood) velocity as

$$
v(x, t)=\frac{\phi(x, t)}{\rho(x, t)} .
$$

yields using (2931) a velocity equation:

$$
\frac{\partial v}{\partial t}=-v+G+\frac{a}{\rho}\left(v \frac{\partial}{\partial x}(\rho G)-\frac{\partial}{\partial x} \rho\right)+\frac{a^{2}}{2 \rho}\left(-v \frac{\partial^{2}}{\partial x^{2}} \rho+\frac{\partial^{2}}{\partial x^{2}}(\rho G)\right) .
$$

We now write $G(x, t)$, the expected update velocity, as a function of $v(x, t)$ and $\rho(x, t)$ only. To simplify we keep only leading order terms in $a$ and obtain

$$
G=(1-2 \eta) \frac{\tanh (\beta[(1-\kappa) v+\kappa \omega])}{\tanh \beta}
$$


where

$$
\omega=\frac{2 a}{3 \rho} \frac{\partial \rho(x, t)}{\partial x} .
$$

Finally, setting $a$ to one in (29131, 34) yields (15]18). 